\title{
Facile Preparation and Characterization of Nanostructured BiOI microspheres with certain adsorption-photocatalytic properties
}

\author{
Yaohui Lv ${ }^{a *}$, Pei Lit $i^{a}$,Yanhao Che ${ }^{a}$, Chao Hü ${ }^{a}$, Songlin Ran ${ }^{b}$, Pingping Shi ${ }^{a}$, Wei Zhang ${ }^{a}$ \\ ${ }^{a}$ School of Materials Science and Engineering, Anhui Key Laboratory of Metal Materials and \\ Processing, Anhui University of Technology, Anhui, Maanshan, 243002, P. R. China \\ ${ }^{b}$ Key Laboratory of Metallurgical Emission Reduction \& Resources Recycling, Anhui University of \\ Technology, Anhui, Maanshan, 243002, P. R. China
}

Received: August 02, 2017; Revised: December 18, 2017; Accepted: February 09, 2018

\begin{abstract}
A novel adsorbent based nanostructured bismuth oxyiodide (BiOI) microspheres combining the highly effective adsorption and green photocatalytic regeneration were fabricated by a facile and rapid low-temperature chemical bath method. The resultant products were characterized by X-ray diffraction (XRD), Scanning electron microscopy (SEM), energy dispersive spectroscopy (EDS), Nitrogen adsorption-desorption measurements, and UV-Vis diffuse reflectance spectroscopy (DRS). The BiOI microspheres displayed remarkable performance towards removal of Rhodamine $\mathrm{B}(\mathrm{RhB})$ and $\mathrm{Cr}$ (VI) from aqueous solution, showing a maximum adsorption capacity reached up to $40.78 \mathrm{mg} \mathrm{g}^{-1}$ and $38.01 \mathrm{mg} \mathrm{g}^{-1}$, respectively. The kinetics and equilibrium of adsorption process were found to follow the pseudo-second-order kinetic and Langmuir isotherm models. Notably, the BiOI microspheres have excellent photocatalytic regeneration capability due to their intrinsically prominent photoresponse in visible light region. By meaning of providing a new insight into the design of new generation adsorbent that could combine adsorption and solar-driven photocatalysis, the synthesized BiOI microspheres are very promising for the large-scale industrial application for water treatment.
\end{abstract}

Keywords: Bismuth oxyiodide, Adsorption, Photocatalysis, Environmental remediation.

\section{Introduction}

Currently, Water quality of global water resources is deteriorating continuously with the development of the industrialization, agriculturalizing as well as civilization activities $^{1}$. The removal of pollutants from contaminated water, such as heavy metals and organic pesticide, is an urgent need for providing disease-free health to our society. For a few decades, considerable effort has been made to develop advanced technology for water purification. Among various technologies, adsorption and photocatalysis are two widely used methodologies to remove organic pollutants from wastewater. Adsorption, as a promising technology, is attractive owing to its high efficiency, good reproducibility, easy handling and low energy requirements. Nevertheless, conventional adsorbents normally suffer from low adsorption capacities and rates, poor separation ability or undesirable regeneration ${ }^{1}$. Since the initial work of and Honda in $1972^{2}$, intense investigation has been dedicated to the photoassisted catalysis over semiconductors for pollutant abatement and waste treatment. Even if photocatalytic processes have shown to be very effective, there are many real situations that they cannot cope with. Therefore, photocatalysis is being combined with physical or chemical operations, which affect the chemical kinetics and/or the overall efficiency is an effective strategy to solve problem of waste treatment ${ }^{3}$. The combinations of photocatalysis may be divided in two main categories: (i) Photocatalysis is coupled with an operation that affects the photocatalytic mechanisms, including ultrasonic irradiation, photo-Fenton reaction, ozonation, electrochemical treatment. (ii) Photocatalysis is coupled with an operation that does not affect the photocatalytic mechanisms, including biological treatment, membrane reactor, membrane photoreactor, physical adsoption ${ }^{4}$. These combinations can increase the efficiency of the photocatalysis, by decreasing the reaction time or the costs 5 .

Among these combination, adsorption-photocatalysis technique is deemed to the most potential strategy for waste treatment ${ }^{6,7}$. In this combination, a suitable adsorbent, such as silica, alumina, zeolites, clays or activated carbon (AC), can selectively adsorbs the toxic pollutants. Once the adsorption reached the saturation state, it is separated from the purified water and mixed with $\mathrm{TiO}_{2}$ powder in water to produce a composite suspension. The desorbing component is photocatalytically oxidized by irradiating the suspension and the adsorbent can be regenerated and reused in further adsorption cycles. Other alternative of photocatalysis-adsorption combination consists in loading the catalyst on the surface of the adsorbent, producing a catalyst-adsorbent system with advantageous features, such as better separability or better aggregation properties. Compared the first combination technique, the advantage point is the adsorption and oxidation of 
organic compounds occur continuously, thus avoiding the need of two independent processes ${ }^{8}$. Recent years have witnessed a growing interest in using the porous natural mineral to support $\mathrm{TiO}_{2}$ nanoparticles. Many studies have focused on the research of fixing $\mathrm{TiO}_{2}$ on zeolite $^{9}$, silica ${ }^{10}$, kaolinite ${ }^{11}$, and other catties. However, owing to its wide band gap $\left(\mathrm{E}_{\mathrm{g}}=3.2 \mathrm{eV}\right), \mathrm{TiO}_{2}$ requires ultraviolet light (UV) instead of more abundant visible light for effective photocatalysis. Therefore, in order to use solar energy efficiency and suit for large-scale industrial applications, many efficient combination adsorption-photocatalysts have been investigated and successfully designed, such as $\mathrm{BiOBr} / \mathrm{Co}-\mathrm{Ni}-\mathrm{NO}_{3}$ layered double hydroxide nanocomposite ${ }^{12}, \mathrm{BiOI} /$ rectorite $^{13}$, G-Fe ${ }_{2} \mathrm{O}_{3} / \mathrm{SiO}_{2}-\mathrm{NH}_{2}{ }^{14}, \mathrm{~g}^{-\mathrm{C}_{3} \mathrm{~N}_{4} / \mathrm{ZnO}^{15}, \mathrm{BiOBr}-\mathrm{PVP}}{ }^{16}, \mathrm{ZIF}-8 /$ $\mathrm{Zn}_{2} \mathrm{GeO}_{4}{ }^{17}$, et al. Recently, new generation adsorbents, single three-dimensional (3D) porous micro/nanoarchitectures, have received increasing interest in environmental remediation ${ }^{18-22}$, which have shown promise for removal of the toxic contaminants from water. For example, Lu et al. ${ }^{23}$ reported the electrochemical method synthesis of hierarchical porous $\mathrm{CeO}_{2}$ nanospheres and demonstrated that such architectures have strong capability towards adsorptive removal Congo Red. Zhang et al. reported the hydrothermal synthesis of layer-structured $\mathrm{Ni}(\mathrm{OH})_{2}$ flower-like architectures exhibiting significantly different selectivities for organic dyes in aqueous solutions ${ }^{24}$. $\mathrm{MoS}_{2}$ nanoflowers prepared by a self-assembly process showed an excellent ability to remove Rhodamine $\mathrm{B}^{25}$. The unique nature of 3D porous hierarchical material, such as high specific surface area and increased interfacial area, will open up a new path for the waste treatment.

Bismuth oxyhalides belong to the family of main group multicomponent metal oxyhalides V-VI-VII, an important class of ternary compound because of its unique and excellent electrical, magnetic, optical and luminescent properties $^{26}$. $\mathrm{BiOX}(\mathrm{X}=\mathrm{Cl}, \mathrm{Br}, \mathrm{I})$ compounds crystallize in the tetragonal matlockite structure, a layer structure characterized by $\left[\mathrm{Bi}_{2} \mathrm{O}_{2}\right]$ slabs interleaved by double slabs of halogen atoms, which results in the general formation of plate morphology $\mathrm{y}^{27}$. Recently, BiOXs have been explored as a novel adsorbent to remove toxic metal ions and organic pollutants from water ${ }^{18,19}$. The aim of this investigation was to provide a new insight into the design of new generation adsorbent that could combine adsorption and solar-driven photocatalysis to meet largescale industrial application for water treatment. The bismuth oxyiodide (BiOI) was reasonably selected due to its best photoresponse among BiOXs. A facile and rapid one-step solution route was applied to construct the hierarchical porous BiOI architectures. The structure, adsorption and regeneration properties of hierarchical porous $\mathrm{BiOI}$ architectures were systematically investigated to evaluate the desirability of their application in water treatment.

\section{Experimental}

\subsection{Synthesis of hierarchical porous BiOI architectures}

Bismuth nitrate pentahydrate $\left(\mathrm{Bi}\left(\mathrm{NO}_{3}\right)_{3} \cdot 5 \mathrm{H}_{2} \mathrm{O}\right)$, potassium iodide (KI) were bought from China National Medicines Co. Ltd. and were used as the Bi and I sources, respectively. All the above analytical chemicals were used without further purification and distilled water was used in all experiments. In a typical procedure, 5 $\mathrm{mmol} \mathrm{Bi}\left(\mathrm{NO}_{3}\right)_{3} \cdot 5 \mathrm{H}_{2} \mathrm{O}$ and $5 \mathrm{mmol}$ of $\mathrm{KI}$ were added into an $100 \mathrm{~mL}$ ethylene glycol solution. The mixture was stirred for $30 \mathrm{~min}$ at room temperature, and then poured into a $250 \mathrm{~mL}$ flask. The flask was heated in oil bath at $160^{\circ} \mathrm{C}$ for $2 \mathrm{~h}$. The resulting products were washed with deionized water and absolute ethanol several times and then dried at $80^{\circ} \mathrm{C}$ for $12 \mathrm{~h}$.

\subsection{Characterization}

The powder X-ray diffraction (XRD) measurements were characterized by using a Bruker D8 advance powder X-ray diffractometer with $\mathrm{Cu} K_{a}$ radiation $(\lambda=0.15405 \mathrm{~nm})$ in the $2 \theta$ range from $20^{\circ}$ to $80^{\circ}$. Field-emission scanning electron microscope (FESEM) images were taken on a Hitachi S-4800 scanning electron microscope. The elemental composition of the sample was characterized by energy-dispersive X-ray spectroscopy (EDS). A nitrogen adsorption system (Micrometritics ASAP2010) was employed to record the adsorption-desorption isotherms at the liquid-nitrogen temperature of $77 \mathrm{~K}$. The Fourier transform infrared (FTIR) spectrum was measured on a Nicolet 6700 FTIR Spectrometric Analyzer using $\mathrm{KBr}$ pellets. UV-Vis diffused reflectance spectra were obtained for the dry-pressed disk samples by a Shimaszu UV 2550 recording spectrophotometer, which was equipped with an integrating sphere, and $\mathrm{BaSO}_{4}$ was used as a reference.

\section{3. $\mathrm{RhB}$ and $\mathrm{Cr}(\mathrm{VI})$ adsorption}

Firstly, aqueous solutions containing different concentrations of RhB (5- $100 \mathrm{mg} / \mathrm{L}$ ) and $\mathrm{Cr}$ (VI) (10$100 \mathrm{mg} / \mathrm{L})$ were prepared. In this case, $\mathrm{K}_{2} \mathrm{Cr}_{2} \mathrm{O}_{7}$ was used as the source of $\mathrm{Cr}$ (VI). For the adsorption rate tests for $\mathrm{RhB}, 50 \mathrm{mg} \mathrm{BiOI}$ adsorbents were added to 100 $\mathrm{mL}$ of $\mathrm{RhB}(50 \mathrm{mg} / \mathrm{L})$ solution and $200 \mathrm{mg}$ adsorbents were added to $100 \mathrm{~mL}$ of $\mathrm{Cr}$ (VI) $(80 \mathrm{mg} / \mathrm{L})$ solution. At predetermined time intervals, solid and liquid were separated by centrifugation of the resulting suspension to measure the concentrations of $\mathrm{RhB}$ and $\mathrm{Cr}$ (VI) in the solution. For adsorption isotherm studies, the $100 \mathrm{~mL}$ of $\mathrm{RhB}$ and $100 \mathrm{~mL}$ of $\mathrm{Cr}$ (VI) solution with different concentrations were mixed with 50 and $200 \mathrm{mg}$ BiOI adsorbents for $90 \mathrm{~min}$. The concentration of $\mathrm{RhB}$ and $\mathrm{Cr}$ (VI) left in the supernatant solution was determined by 
using a Shimadzu UV 2550 UV-vis spectrophotometer. The RhB concentration was determined by measuring the solution absorbance at $554 \mathrm{~nm}$. The characteristic absorption of $\mathrm{Cr}(\mathrm{VI})$ at around $354 \mathrm{~nm}$ was used to monitor the $\mathrm{Cr}$ (VI) concentration. The UV-visible absorbance of aqueous $\mathrm{RhB}$ and $\mathrm{Cr}$ (VI) solution as a function of the aqueous solution concentration was shown in Supporting information Figure S1 and Figure S2, which it exhibit good liner behavior. For reference, the carbon spheres were selected to compare the adsorption capacity to that to the hierarchical BiOI microspheres. The detailed preparation process of carbon spheres and SEM images was shown in Supporting information Figure S3.

\subsection{Photocatalytically recycling under visible light}

The regeneration experiments were conducted in the $100 \mathrm{~mL}$ of $50 \mathrm{mg} / \mathrm{L} \mathrm{RhB}$ solution with $50 \mathrm{mg}$ BiOI hierarchical microspheres at room temperature for 90 $\mathrm{min}$. The $\mathrm{CR}$-adsorbed $\mathrm{BiOI}$ is collected through natural settlement for $30 \mathrm{~min}$, dispersed in $200 \mathrm{~mL}$ distilled water, irradiated under a $300 \mathrm{~W}$ Xenon lamp through a UV-cutoff filter $(420 \mathrm{~nm})$ for $1 \mathrm{~h}$, and then reused for adsorption again. The distance between it and the photocatalyst was $50 \mathrm{~cm}$. The light intensity reaching the samples was measured using a radiometer and was found to be approximately $35 \mathrm{~W} \mathrm{~m}^{-2}$ in the visible-light range. The supernatant solutions were analyzed by UV-vis spectroscopy. Similarly, the $100 \mathrm{~mL}$ of $80 \mathrm{mg} / \mathrm{L} \mathrm{Cr}$ (VI) solution with $200 \mathrm{mg}$ BiOI hierarchical microspheres was applied to conduct the regeneration experiments.

\section{Results and Discussion}

\subsection{Structure and morphology of hierarchical porous BiOI architectures}

The phase structure of the as-prepared BiOI product was examined by X-ray diffraction (XRD). Figure 1 depicts the XRD patterns of the hierarchical BiOI flowerlike sphere before and after adsorption. It can be found that the XRD patterns of hierarchical BiOI flower-like spheres before and after adsorption present similar profiles. The diffraction peaks situated at $29.5^{\circ}, 31.7^{\circ}$, $33.3^{\circ}, 37.1^{\circ}, 45.4^{\circ}, 51.1^{\circ}, 55.3^{\circ}$ and $66.4^{\circ}$ are observed, which are due to the BiOI (012), (110), (111), (013), (014), (114), (122) and (220) lattice planes, respectively, which indicating the sample is the pure the tetragonal phase of BiOI (JCPDS file No. 73-2062). No detection of other crystalline impurities suggests that the product is high purity and single phase. It is noteworthy that the crystal structure was not changed after the adsorption. The XRD result indicates that the BiOI hierarchical microspheres are stable during the adsorption process.

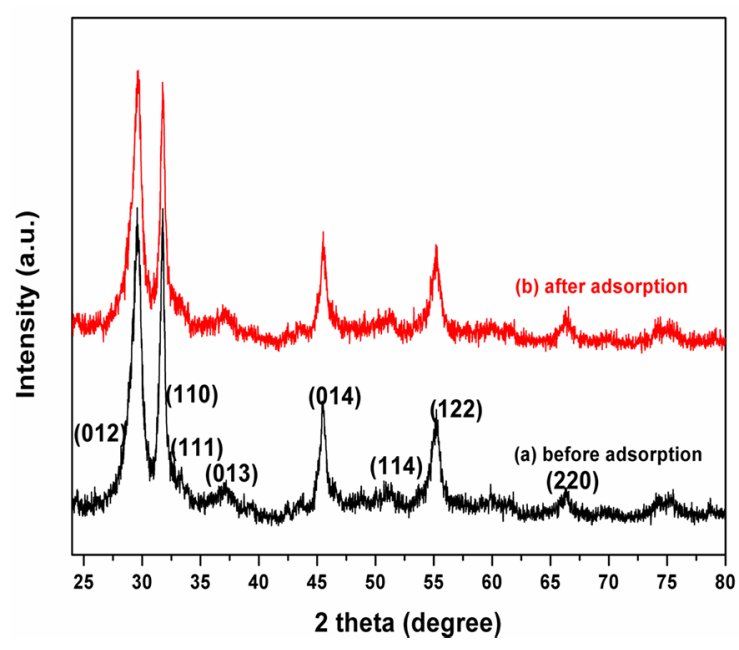

Figure 1. XRD patterns of the BiOI hierarchical structure before and after RhB adsorption

The morphology of the products was determined by the field-emission scanning electron microscopy (FESEM). Figure 2a is a typical FESEM image of the $\mathrm{BiOI}$ hierarchical microspheres. It reveals that the flowerlike superstructures are composed of nanosheets with thickness of $20 \mathrm{~nm}$, forming an open porous structure. The composition of the product was determined by $\mathrm{X}$-ray energy-dispersive spectroscopy (EDS), as shown in Figure $2 b$, which unambiguously demonstrates the coexistence of $\mathrm{Bi}, \mathrm{O}$ and I elements in the product.

\subsection{Specific surface area and porosity}

The specific surface area and porosity of the BiOI microspheres were further evaluated by nitrogen adsorption-desorption isotherm measurement. Figure 3 shows the nitrogen adsorption-desorption isotherms and pore size distributions of the BiOI microspheres. The Brunauer-Emmett-Teller (BET) surface area was calculated to be $38.1 \mathrm{~m}^{2} \mathrm{~g}^{-1}$. As shown in Figure 3, the assynthesized BiOI microsphere exhibit type-IV isotherms with hysteresis loops at relative pressures between 0.5 and 1.0 , which is possibly associated with the existence of mesopores with different pore size distributions in the microspheres. In addition, the pore diameters are estimated by using the BJH method. As shown in the insets, the $\mathrm{BiOI}$ microspheres possess an average pore diameter of $25.2 \mathrm{~nm}$. Owing to the specific surface area and strong adsorption (great hysteresis of the desorption isotherm), the nanostructured BiOI microsphere should have excellent good ion adsorption properties.

\subsection{Surface properties and stability}

In order to investigate the adsorption process between $\mathrm{RhB}$ and BiOI, the FTIR spectroscopic analysis of the 


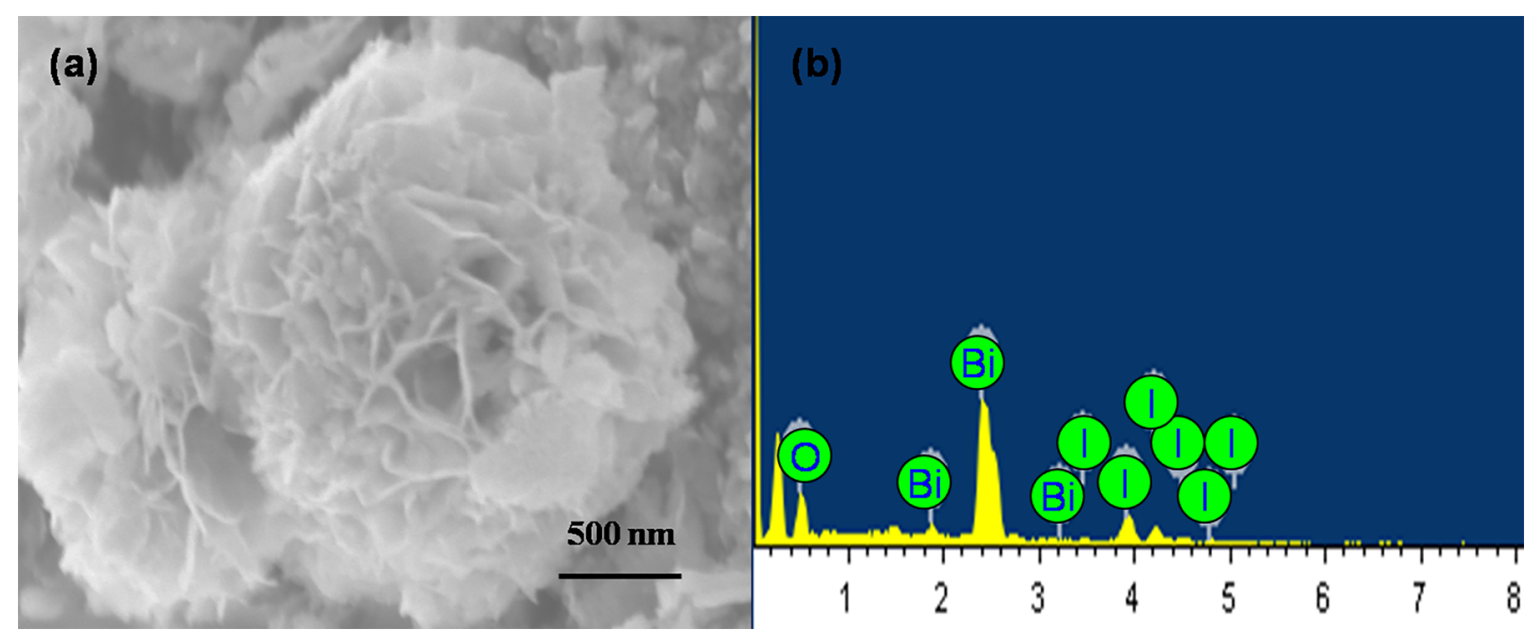

Figure 2. SEM image (a) and EDS spectrum (b) of the BiOI hierarchical structure

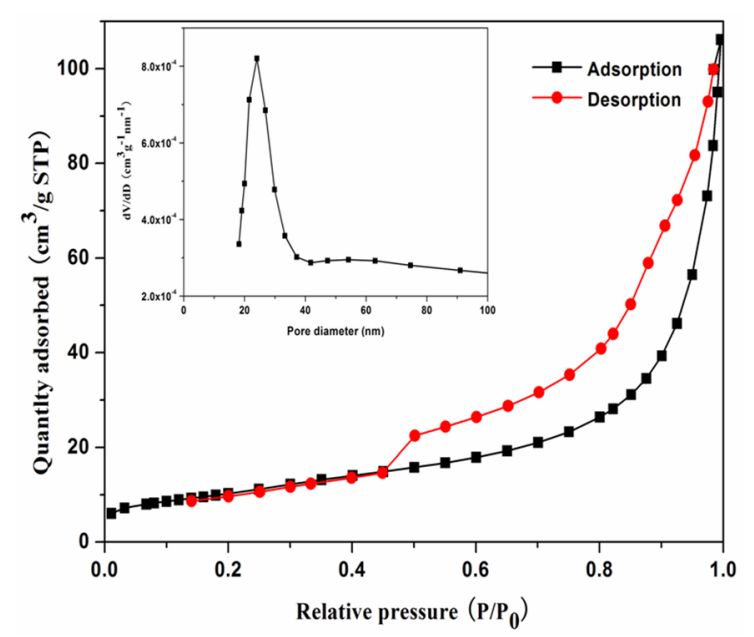

Figure 3. Nitrogen adsorption-desorption isotherms and pore size distributions (insets) of $\mathrm{BiOI}$ microspheres

BiOI hierarchical architectures before and after the $\mathrm{RhB}$ adsorption was characterized, as shown in Figure 4a. It can be clearly seen that the absorption bands in the range of $800-1500 \mathrm{~cm}^{-1}$ assigned to the RhB molecules could be clearly seen in the FTIR spectrum of the RhB-adsorbed $\mathrm{BiOI}$ architectures. Compared to the as-prepared BiOI, there are several obvious new bands in the spectrum of $\mathrm{RhB}$ saturated BiOI, such as the bands at around 1413, 1176 and $1131 \mathrm{~cm}^{-1}$, corresponding to the stretching vibration of $-\mathrm{C}=\mathrm{C}-$, the stretching vibration of $-\mathrm{C}-\mathrm{C}-$, and the stretching vibration of -C-O-C-, respectively. The above result validated the $\mathrm{RhB}$ dyes were anchored on the surface of the BiOI architectures during the adsorption process. Figure $4 \mathrm{~b}$ shows a typical SEM image of the RhB-adsorbed BiOI architectures, which exhibits similar morphology to that of the fresh BiOI architectures. Combining with the XRD results, the BiOI architectures are stable during the adsorption process, which is important for its practical application.

\subsection{Adsorption of $R h B$ dye}

Figure.5a represents the time-dependent $\mathrm{RhB}$ dye concentration variation curve over different adsorbents with an initial dye concentration of $50 \mathrm{mg} \mathrm{L}^{-1}$, respectively. Compared with commercial $\mathrm{TiO}_{2}$ and as-synthesized carbon sphere, the flower-like BiOI nanostructures demonstrate highly efficient RhB dye adsorptive capacities. The adsorption is very fast within the first $15 \mathrm{~min}$, and reaches adsorption-desorption equilibrium after $60 \mathrm{~min}$. This high adsorption efficiency can be ascribed to abundant unoccupied active sites on the surface of the adsorbent, the high dye concentration gradient at the initial stage, as well as the high specific surface area of the BiOI architectures. The above adsorption experiment data were further fitted by the pseudo-first-order and pseudo-second-order kinetic models to obtain the characteristics of the adsorption process. Two kinetic models are expressed as shown in Eqs. (1) and (2), respectively ${ }^{28,29}$.

$$
\begin{gathered}
\ln \left(q_{e}-q_{t}\right)=\ln q_{e}-K_{1} t \\
t / q_{t}=\left(K_{2} q_{e}^{2}\right)^{-1}+t / q_{e}
\end{gathered}
$$

Where $\mathrm{q}_{\mathrm{t}}\left(\mathrm{mg} \mathrm{g}^{-1}\right)$ is the amounts of RhB adsorbed at any time $\mathrm{t}(\mathrm{min}), \mathrm{q}_{\mathrm{e}}$ is the equilibrium adsorption capacity $\left(\mathrm{mg} \mathrm{g}^{-1}\right), K_{1}\left(\mathrm{~min}^{-1}\right)$ and $K_{2}\left(\mathrm{~g} \mathrm{mg}^{-1} \mathrm{~min}^{-1}\right)$ are the rate constants of pseudo-first-order and paeudo-second-order adsorption, respectively. All kinetic parameters and the correlation coefficients were calculated by linear regression (Figure S4) and given in Table 1. The relatively high correlation coefficients $\left(\mathrm{R}^{2}\right)$ of the pseudo-second-order model and the small difference between the calculated value of adsorption capacity $\left(q_{e}, c a l\right)$ and the experimental value $\left(q_{e}, \exp \right)$ indicate that the adsorption kinetics of $\mathrm{RhB}$ on 

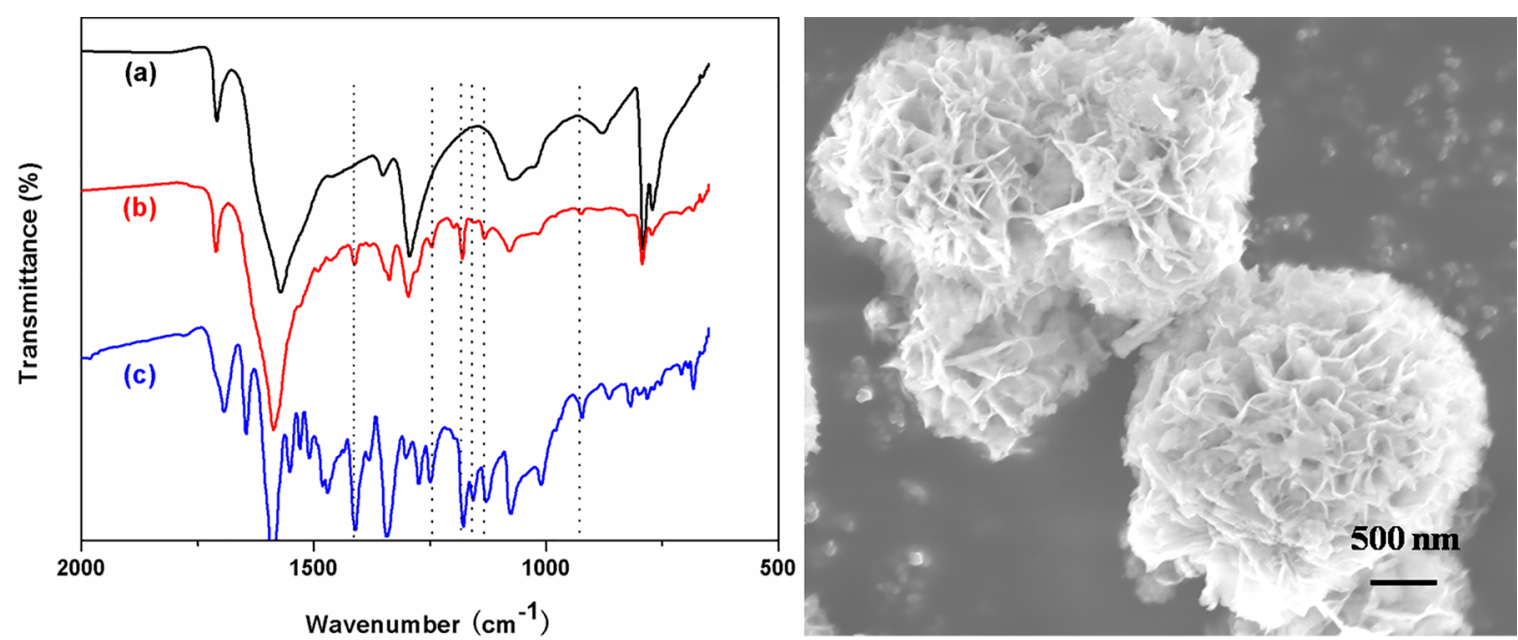

Figure 4. FTIR spectra of the BiOI architectures before (a) and after (b) RhB adsorption and (c) pure RhB (left) and SEM image of $\mathrm{RhB}$-adsorbed $\mathrm{BiOI}$ hierarchical structure (right)
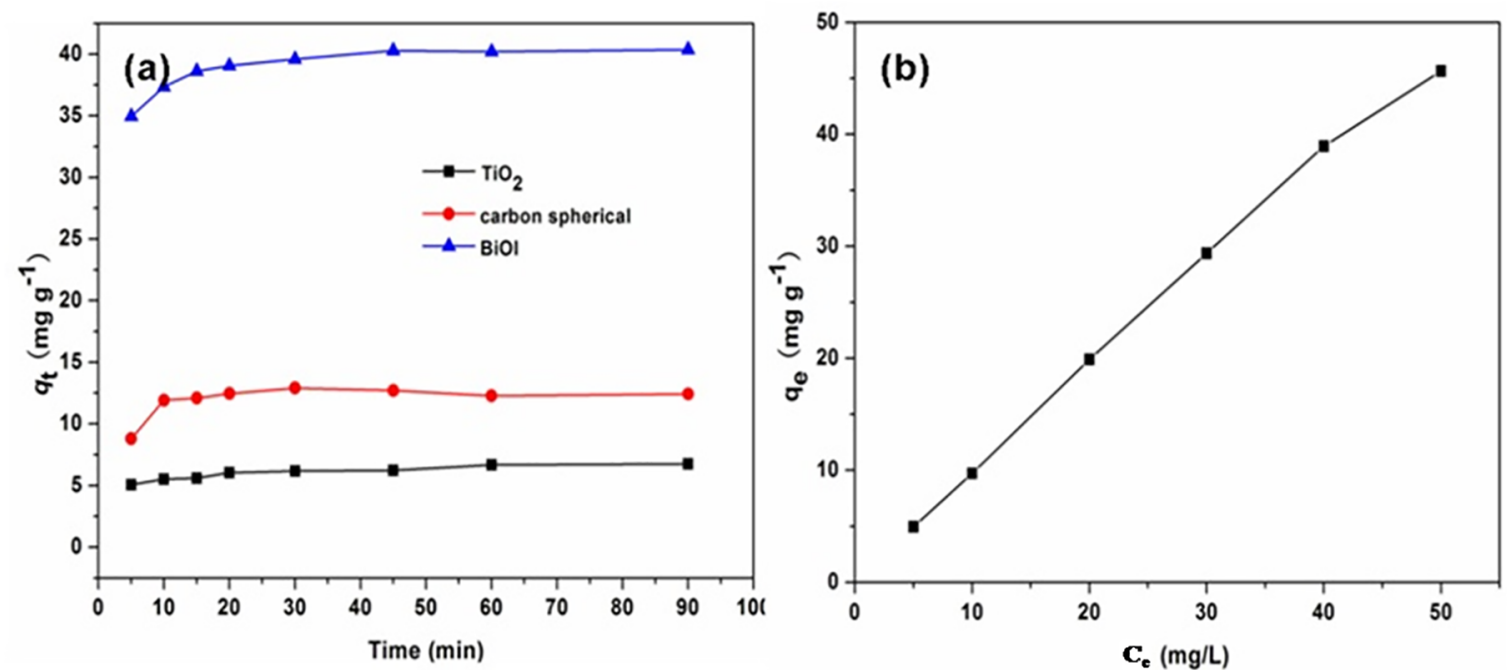

Figure 5. Time-dependent $\mathrm{RhB}$ concentration variation curve (a) and adsorption isotherms (b) over flower-like BiOI microspheres

Table 1. Kinetic parameters for the adsorption of $\mathrm{RhB}$ onto BiOI sample

\begin{tabular}{lccccccc}
\hline Sample & $\boldsymbol{q}_{e}, \exp (\mathrm{mg} / \mathrm{g})$ & \multicolumn{3}{c}{ First-order kinetic model } & \multicolumn{3}{c}{ Second-order kinetic model } \\
\hline & & $K_{1}\left(\mathrm{~min}^{-1}\right)$ & $R^{2}$ & $q_{e, \text { cal }}(\mathrm{mg} / \mathrm{g})$ & $K_{2}(\mathrm{~g} /(\mathrm{mg} \cdot \mathrm{h}))$ & $R^{2}$ & $q_{e, \text { cal }}(\mathrm{mg} / \mathrm{g})$ \\
BiOI & 40.37 & 0.0722 & 0.85818 & 5.79 & 0.0292 & 0.99998 & 40.78 \\
\hline
\end{tabular}

the BiOI microspheres obey the pseudo-second-order kinetic model. The result indicates that the adsorption process is mainly controlled by chemisorption.

To further investigate the adsorption, the adsorption isotherm of $\mathrm{RhB}$ on the as-prepared $\mathrm{BiOI}$ microspheres was also measured under different initial concentrations, as shown in Figure. 5b. The adsorption capacity increases with increasing initial dye concentration and the Langmuir ${ }^{30}$ and Freundlich ${ }^{31}$ adsorption models were used to analyse the equilibrium adsorption characteristics.

$$
C_{e} / q_{e}=\left(q_{\text {emax }} K_{L}\right)^{-1}+C_{e} / q_{\max }
$$

$$
\ln q_{e}=\ln K_{F}+1 / n \ln C_{e}
$$

where $C_{e}$ is the equilibrium concentration of the $\mathrm{RhB}$ dye $\left(\mathrm{mg} \mathrm{L}^{-1}\right) ; q_{e}$ is the equilibrium adsorption capacity ( $\mathrm{mg}$ $\left.\mathrm{g}^{-1}\right) ; q_{e \max }$ and $K_{L}$ are constants related to the maximum adsorption amount of RhB adsorbed per unit weight of adsorbent ( $\left.\mathrm{mg} \mathrm{g}^{-1}\right)$ and the energy of adsorption $\left(\mathrm{L} \mathrm{mg}^{-1}\right)$, respectively; $K_{F}$ is roughly an indicator of the adsorption capacity, and $1 / n$ is the adsorption intensity. The relative parameters obtained from liner regression by the two models were calculated (Figure S5) and shown in Table 2. Obviously, the Langmuir model $\left(\mathrm{R}^{2}=0.95838\right)$ gave a better 
fit than the Freundlich model $\left(\mathrm{R}^{2}=0.73824\right)$ according to their correlation coefficients. The result indicates that the $\mathrm{RhB}$ dye adsorb as a monolayer coverage on the BiOI microsphere surface. This result is in good agreement with the observations in previous on the adsorption properties of hierarchical architectures ${ }^{19,32}$.

\section{5. $\mathrm{Cr}$ (VI) removal capacity}

The chromium (VI) ion is a toxic heavy metal ion typically found in water resources that is harmful to human health. Figure. 6 shows the adsorption rate and capacity of nanostructured BiOI microspheres for a $\mathrm{Cr}$ (VI) solution. The adsorption is very fast within the first $30 \mathrm{~min}$, and reaches adsorption-desorption equilibrium after $90 \mathrm{~min}$ (Figure. 6a). All kinetic parameters and the correlation coefficients were calculated by linear regression (Figure S6) and given in Table 3. The results show that the adsorption kinetics of $\mathrm{RhB}$ on the $\mathrm{BiOI}$ microspheres obeys the pseudo-second-order kinetic model.
To further investigate the adsorption, the adsorption isotherm of $\mathrm{Cr}(\mathrm{VI})$ on the as-prepared $\mathrm{BiOI}$ microspheres was also measured under different initial concentrations, as shown in Figure. 6b. The relative parameters obtained from liner regression by the two models were calculated (Figure S7) and shown in Table 4. Apparently, the experimental data fit better to the Langmuir adsorption model than to the Freundlich isotherm, which indicates that the $\mathrm{Cr}(\mathrm{VI})$ ions adsorb as a monolayer coverage on the BiOI microsphere surface. From the standard equation of the Langmuir model, the $\mathrm{Cr}(\mathrm{VI})$ removal capacity of $\mathrm{BiOI}$ microspheres is calculated to be $38.01 \mathrm{mg} \mathrm{g}^{-1}$.

\subsection{Photocatalytic regeneration capacity}

Recently, photocatalysis technology was widely used to remove organic pollutants from wastewater. Therefore, we believe the RhB-adsorbed BiOI microspheres could be regenerated via the economical, green photocatalytic treatment. The optical properties
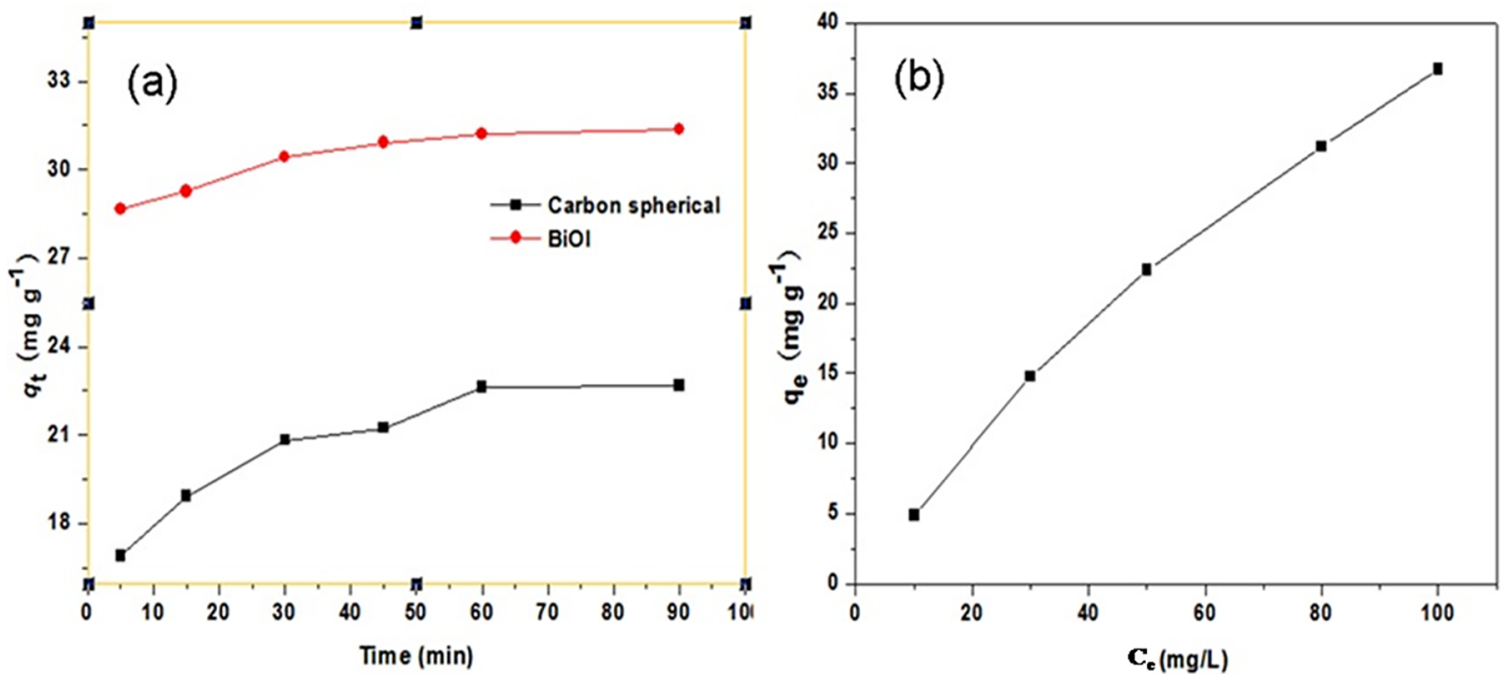

Figure 6. Time-dependent $\mathrm{Cr}$ (VI) concentration variation curve (a) and adsorption isotherms (b) over flower-like BiOI microspheres

Table 2. Isotherm parameters for the adsorption of $\mathrm{RhB}$ onto BiOI sample

\begin{tabular}{lcccccc}
\hline Sample & \multicolumn{3}{c}{ Langmuir model } & \multicolumn{3}{c}{ Freundlich model } \\
\hline & $K(\mathrm{~L} / \mathrm{mg})$ & $R^{2}$ & $q \max (\mathrm{mg} / \mathrm{g})$ & $\begin{array}{c}K_{F}[(\mathrm{mg} / \mathrm{g})(\mathrm{L} / \\
\left.\mathrm{mg})^{1 / \mathrm{n}}\right]\end{array}$ & $R^{2}$ & $n$ \\
BiOI & 2.0342 & 0.95838 & 50.94 & 29.383 & 0.73824 & 2.2885 \\
\hline
\end{tabular}

Table 3. Kinetic parameters for the adsorption of $\mathrm{Cr}(\mathrm{VI})$ onto BiOI sample

\begin{tabular}{lccccccc}
\hline Sample & qe,exp $(\mathrm{mg} / \mathrm{g})$ & \multicolumn{3}{c}{ First-order kinetic model } & \multicolumn{2}{c}{ Second-order kinetic model } \\
\hline & & $k_{1}\left(\mathrm{~min}^{-1}\right)$ & $R^{2}$ & $\mathrm{q}_{\mathrm{e}, \mathrm{cal}}(\mathrm{mg} / \mathrm{g})$ & $k_{2}(\mathrm{~g} /(\mathrm{mg} \cdot \mathrm{h}))$ & $R^{2}$ & $\mathrm{q}_{\mathrm{e}, \mathrm{cal}}(\mathrm{mg} / \mathrm{g})$ \\
BiOI & 31.35 & 0.0538 & 0.98343 & 4.20 & 0.03311 & 0.99992 & 31.66 \\
\hline
\end{tabular}

Table 4. Isotherm parameters for the adsorption of $\mathrm{Cr}(\mathrm{VI})$ onto BiOI sample

\begin{tabular}{lcccccc}
\hline Sample & \multicolumn{3}{c}{ Langmuir model } & \multicolumn{3}{c}{ Freundlich model } \\
\hline & $K(\mathrm{~L} / \mathrm{mg})$ & $R^{2}$ & $\mathrm{q}_{\max }(\mathrm{mg} / \mathrm{g})$ & $\begin{array}{c}K_{\mathrm{f}}[(\mathrm{mg} / \mathrm{g})(\mathrm{L} / \\
\left.\mathrm{mg})^{1 / \mathrm{n}}\right]\end{array}$ & $R^{2}$ & $\mathrm{n}$ \\
BiOI & 0.4471 & 0.9842 & 38.01 & 11.8054 & 0.82462 & 2.8026 \\
\hline
\end{tabular}


of the BiOI microspheres were investigated by UV-Vis diffuse reflectance spectroscopy. As shown in Figure 7, the BiOI microspheres display the strong absorption ranged from 200 to $650 \mathrm{~nm}$. The band gap energy of the BiOI microspheres is estimated to be $1.71 \mathrm{eV}$, which is similar to the reported hierarchical BiOI architectures ${ }^{33,34}$. Therefore, the photocatalytic regeneration property of the BiOI microspheres was evaluated under visible light. Figure. 8 shows the successive cycles of the adsorptionregeneration process. The BiOI microspheres can be recycled and reused for three successive cycles with a removal efficiency of around $74 \%$. It is clear that a decrease in the removal efficiency was observed when the BiOI microspheres were reused for the first cycle. The decreased removal efficiency after three cycles may be due to the obvious mass loss during the sedimentation and transferring process, and the decreased surface active sites.

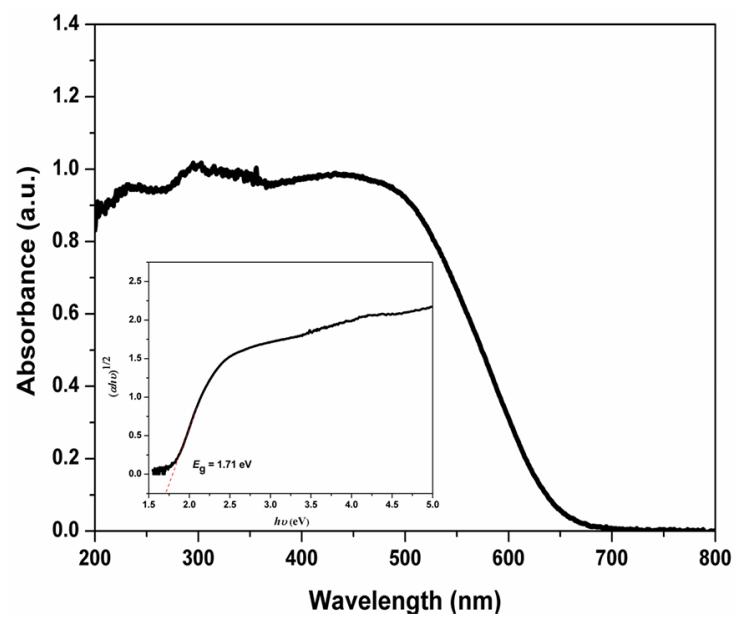

Figure 7. UV-vis diffuse reflectance spectroscopy of the BiOI hierarchical structure; inset in figure shows the plots of $(\alpha h v)^{1 / 2}$ vs. photon energy $(\mathrm{h} v)$ for the BiOI hierarchical structure

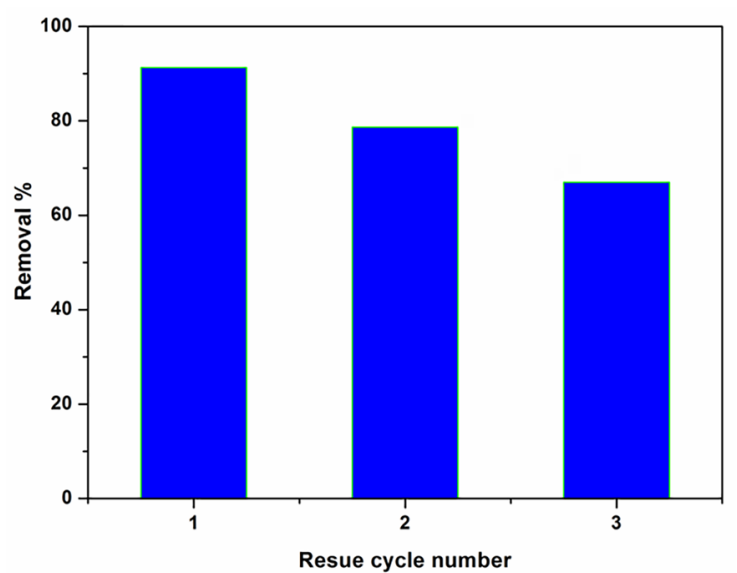

Figure 8. Recycling test of the RhB-adsorbed BiOI microspheres performed on adsorption-regeneration process

\section{Conclusions}

In summary, the nanostructured BiOI microspheres have been successfully prepared via a facile and rapid lowtemperature chemical bath method. Experiments indicated that the $\mathrm{BiOI}$ microspheres exhibited superior performance for adsorptive removal of $\mathrm{RhB}$ and $\mathrm{Cr}(\mathrm{VI})$. Specifically, the BiOI microspheres can be easily economically regenerated, which is desirable for practice application. We believe that the new generation adsorbent combining adsorption with solar-driven photocatalysis would be applied as a promising absorbent in large-scale industrial water treatment.

\section{Acknowledgements}

This research was financially supported by the Natural Science Foundation of AnHui Provincial Education Department (KJ2016A102), Graduate student Innovation Fund of Anhui University of Technology and National Undergraduate Training Programs for Innovation and Entrepreneurship (201510360012)

\section{References}

1. Ai L, Zeng Y, Jiang J. Hierarchical porous BiOI architectures: Facile microwave nonaqueous synthesis, characterization and application in the removal of Congo red from aqueous solution. Chemical Engineering Journal. 2014;235(1):331-339.

2. Fujishima A, Honda K. Electrochemical photolysis of water at a semiconductor electrode. Nature. 1972;238(5358):37-38.

3. Zhang YY, Sun XG, Yang GZ, Zhu YH, Si HY, Zhang JM, et al. Preparation and characterization of bifunctional $\mathrm{BiOCl}_{x} \mathrm{I}_{y}$ solid solutions with excellent adsorption and photocatalytic abilities for removal of organic dyes. Materials Science in Semiconductor Processing. 2006;41:193-199.

4. Augugliaro V, Litter M, Palmisano L, Soria J. The combination of heterogeneous photocatalysis with chemical and physical operations: A tool for improving the photoprocess performance. Journal of Photochemistry and Photobiology C: Photochemistry Reviews. 2006;7(4):127-144.

5. Gu YY, Zhao L, Yang MY, Xiong YQ, Wu Z, Zhou MJ, et al. Preparation and characterization of highly photocatalytic active hierarchical $\mathrm{BiOX}(\mathrm{X}=\mathrm{Cl}, \mathrm{Br}, \mathrm{I})$ microflowers for rhodamine $\mathrm{B}$ degradation with kinetic modelling studies. Journal of Central South University. 2017;24(4):754-765.

6. Chang MJ, Wang H, Liu J, Du HL, Li HL. Facile Synthesis of $\mathrm{Fe}_{3} \mathrm{O}_{4} @$ BiOI Core/Shell Nanostructres by Magenetic-Assisted Successive Ionic Layer Adsorption and Reaction for Catalytic Application. Journal of Nanoscience and Nanotechnology. 2017;17(6):3759-3764.

7. Li WT, Xiao F, Su H, Wang DS, Yang XF. Investigation of adsorption and photocatalytic activities of in situ cetyltrimethlammonium bromide-modified $\mathrm{Bi} / \mathrm{BiOCl}$ heterojunction photocatalyst for organic contaminants removal. RSC Advances. 2016;6(96):9330993317. 
8. Albuquerque R, Neves MC, Mendonça MH, Trindade T, Monteiro OC. Adsorption and catalytic properties of $\mathrm{SiO}_{2} / \mathrm{Bi}_{2} \mathrm{~S}_{3}$ nanocomposites on the methylene blue photodecolorization process. Colloids and Surfaces A: Physicochemical and Engineering Aspects. 2008;328(1-3):107-113.

9. Sun Q, Hu XL, Zheng SL, Sun ZM, Liu SS, Li H. Influence of calcination temperature on the structural, adsorption and photocatalytic properties of $\mathrm{TiO}_{2}$ nanoparticles supported on natural zeolite. Powder Technology. 2015;274:88-97.

10. Castañeda-Contreras J, Marañón-Ruiz VF, Chiu-Zárate R, Pérez-Ladrón de Guevara H, Rodriguez R, Michel-Uribe C. Photocatalytic activity of erbium-doped $\mathrm{TiO}_{2}$ nanoparticles immobilized in macro-porous silica films. Materials Research Bulletin. 2012;47(2):290-295.

11. Mamulová Kutláková K, Tokarský J, Kovář P, Vojtěšková S, Kovářová A, Smetana B, et al. Preparation and characterization of photoactive composite kaolinite/ $\mathrm{TiO}_{2}$. Journal of Hazardous Materials. 2011;188(1-3):212-220.

12. Ao YH, Wang DD, Wang PF, Wang C, Hou J, Qian J. A BiOBr/ Co-Ni layered double hydroxide nanocomposite with excellent adsorption and photocatalytic properties. RSC Advances. 2015;5(67):54613-54621.

13. Wu SX, Fang JZ, Hong XT, Hui KS, Chen YF. Facile preparation and characterization of BiOI-rectorite composite with high adsorptive capacity and photocatalytic activity. Dalton Transactions. 2014;43(6):2611-2619.

14. Yang XL, Wang XY, Liu XZ, Zhang YJ, Song WG, Shu CY, et al. Preparation of graphene-like iron oxide nanofilm/silica composite with enhanced adsorption and efficient photocatalytic properties. Journal of Materials Chemistry A . 2013;1(29):83328337.

15. Li XF, Li M, Yang JH, Li XY, Hu TJ, Wang JS, et al. Synergistic effect of efficient adsorption $\mathrm{g}-\mathrm{C}_{3} \mathrm{~N}_{4} / \mathrm{ZnO}$ composite for photocatalytic property. Journal of Physics and Chemistry of Solids. 2014;75(3):441-446.

16. Li YQ, Wang ZY, Huang BB, Dai Y, Zhang XY, Qin XY. Synthesis of BiOBr-PVP hybrids with enhanced adsorption-photocatalytic properties. Applied Surface Science. 2015;347:258-264.

17. Liu Q, Low ZX, Li LX, Razmjou A, Wang K, Yao JF, et al. ZIF-8/ $\mathrm{Zn}_{2} \mathrm{GeO}_{4}$ nanorods with an enhanced $\mathrm{CO}_{2}$ adsorption property in an aqueous medium for photocatalytic synthesis of liquid fuel. Journal of Materials Chemistry A. 2013;1(38):11563-11569.

18. Li GF, Qin F, Wang RM, Xiao SQ, Sun HZ, Chen R. BiOX $(\mathrm{X}=\mathrm{Cl}, \mathrm{Br}, \mathrm{I})$ nanostructures: mannitol-mediated microwave synthesis, visible light photocatalytic performance, and $\mathrm{Cr}(\mathrm{VI})$ removal capacity. Journal of Colloid and Interface Science. 2013;409:43-51.

19. Wang XQ, Liu WX, Tian J, Zhao ZH, Hao P, Kang XL, et al. $\mathrm{Cr}(\mathrm{VI}), \mathrm{Pb}(\mathrm{II}), \mathrm{Cd}(\mathrm{II})$ adsorption properties of nanostructured $\mathrm{BiOBr}$ microspheres and their application in a continuous filtering removal device for heavy metal ions. Journal of Materials Chemistry A. 2014;2(8):2599-2608.

20. Zhang DQ, Wen MC, Jiang B, Li GS, Yu JC. Ionothermal synthesis of hierarchical $\mathrm{BiOBr}$ microspheres for water treatment. Journal of Hazardous Materials. 2012;211-212:104-111.
21. Song LM, Zhang SJ, Wei QW. Porous BiOI Sonocatalysts: Hydrothermal Synthesis, Characterization, Sonocatalytic and Kinetic Properties. Industrial \& Engineering Chemistry Research. 2012;51(3):1193-1197.

22. Chen L, He S, He BY, Wang TJ, Su CL, Zhang C, et al. Synthesis of Iron-Doped Titanium Oxide Nanoadsorbent and Its Adsorption Characteristics for Fluoride in Drinking Water. Industrial \& Engineering Chemistry Research. 2012;51(40):13150-13156.

23. Ouyang XW, Li W, Xie SL, Zhai T, Yu MH, Gan JY, et al. Hierarchical $\mathrm{CeO}_{2}$ nanospheres as a high-efficient adsorbent for dyes removal. New Journal of Chemistry. 2013;37(3):585-588.

24. Ran SH, Zhu YG, Hang HT, Liang B, Xu J, Liu B, et al. Phasecontrolled synthesis of $3 \mathrm{D}$ flower-like $\mathrm{Ni}(\mathrm{OH})_{2}$, architectures and their applications in water treatment. CrystEngComm. 2012;14(9):3063-3068.

25. Wang XH, Ding JJ, Yao SW, Wu XX, Feng QQ, Wang ZH, et al. High supercapacitor and adsorption behaviors of flowerlike $\mathrm{MoS}_{2}$ nanostructures. Journal of Materials Chemistry A. 2014;2(38):15958-15963.

26. Lv YH, Liu H, Zhang W, Ran SL, Chi FL, Yang B, et al. Room-temperature synthesis and high visible-light-induced photocatalytic activity of AgI/BiOI composites. Journal of Environmental Chemical Engineering. 2013;1(3):526-533.

27. Xiao X, Zhang WD. Facile synthesis of nanostructured BiOI microspheres with high visible light-induced photocatalytic activity. Journal of Materials Chemistry. 2010;20(28):58665870 .

28. Lagergren S. About the theory of so-called adsorption of soluble substances. Kungliga Svenska Vetenskapsakademiens Handlingar. 1898;24(4):1-39.

29. Ho YS, McKay G. Sorption of dye from aqueous solution by peat. Chemical Engineering Journal. 1998;70(2):115-124.

30. Sheng PX, Ting YP, Chen JP, Hong L. Sorption of lead, copper, cadmium, zinc, and nickel by marine algal biomass: characterization of biosorptive capacity and investigation of mechanisms. Journal of Colloid and Interface Science. 2004;275(1):131-141

31. Reddad Z, Gerente C, Andres Y, Le Cloirec P. Adsorption of Several Metal Ions onto a Low-Cost Biosorbent: Kinetic and Equilibrium Studies. Environmental Science \& Technology. 2002;36(9):2067-2073.

32. Zhang L, Cao XF, Chen XT, Xue ZL. BiOBr hierarchical micropheres: Microwave-assisted solvothermal synthesis, strong adsorption and excellent photocatalytic properties. Journal of Colloid and Interface Science. 2011;354(2):630-636.

33. Shi X, Chen X, Chen X, Zhou S, Lou S. Solvothermal synthesis of BiOI hierarchical spheres with homogeneous sizes and their high photocatalytic performance. Materials Letters. 2012;68:296-299.

34. Xia J, Yin S, Li H, Xu H, Xu L, Zhang Q. Enhanced photocatalytic activity of bismuth oxyiodine (BiOI) porous microspheres synthesized via reactable ionic liquid-assisted solvothermal method. Colloids and Surfaces A: Physicochemical and Engineering Aspects. 2011;387(1-3):23-28. 


\section{Suplementary material}

The following online material is available for this article:

Figure S1. UV-visible absorbance of aqueous $\mathrm{Cr}$ (VI) solution as a function of the aqueous $\mathrm{Cr}$ (VI) concentration.

Figure S2. UV-visible absorbance of aqueous $\mathrm{Cr}$ (VI) solution as a function of the aqueous $\mathrm{Cr}$ (VI) concentration.

Figure S3. FESEM images of synthesized carbon spheres via hydrothermal reaction

Figure S4. Pseudo-first-order (right) and pesudo-second-order (left) kinetics plots of RhB adsorption onto BiOI product

Figure S5. Adsorption isotherm curves for the adsorption of RhB onto BiOI product. (a) Freundlich mode; (b) Langmuir mode

Figure S6. Pseudo-first-order (a) and pesudo-second-order (b) kinetics plots of RhB adsorption onto BiOI product

Figure S7. Adsorption isotherm curves for the adsorption of $\mathrm{Cr}(\mathrm{VI})$ onto BiOI product. (a) Freundlich mode; (b) Langmuir mode 